\title{
On Ulam-von Neumann Transformations
}

\author{
Yunping Jiang ${ }^{1}$ \\ Department of Mathematics, Queens College of CUNY, Flushing, NY 11367, USA
}

Received: 15 November 1993/in revised form: 8 February 1995

\begin{abstract}
We define and study Ulam-von Neumann transformations which are certain interval mappings and conjugate to $q(x)=1-2 x^{2}$ on $[-1,1]$. We use a singular metric on $[-1,1]$ to study a Ulam-von Neumann transformation. This singular metric is universal in the sense that it does not depend on any particular mapping but only on the exponent of this mapping at its unique critical point. We give the smooth classification of Ulam-von Neumann transformations by their eigenvalues at periodic points and exponents and asymmetries.
\end{abstract}

\section{Contents}

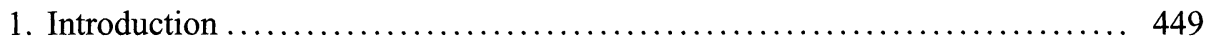

2. Singular change of metric on an interval .......................... 451

3. Ulam-von Neumann transformations............................. 452

4. Complete smooth invariants ................................. 454

\section{Introduction}

Ulam and von Neumann studied the chaotic behavior of the nonlinear self mapping $q(x)=1-2 x^{2}$ of the interval $[-1,1]$ in 1947. They observed that $\rho_{q}=$ $1 /\left(\pi \sqrt{1-x^{2}}\right)$ is the density function of a unique absolutely continuous $q$-invariant measure (we only consider probability measures). In modern language, this observation shows that $q$ is a chaotic dynamical system and follows from making the singular change of metric $d y=(2 / \pi)\left(d x / \sqrt{1-x^{2}}\right)$. If we let $y=h(x)$ be the corresponding change of coordinate and $\tilde{q}=h \circ q \circ h^{-1}$, then $q$ becomes $\tilde{q}(y)=1-2|y|$, a piecewise linear mapping with expansion rate 2 on $[-1,1]$. The dynamics of $\tilde{q}$ is more easily understood.

Now consider a general self mapping $f$ of $[-1,1]$ whose graph looks like those in Fig. 1. Then $f$ is topologically conjugate to $q$ under a certain smoothness

\footnotetext{
1 The author is partially supported by a PSC-CUNY grant and a NSF grant.
} 


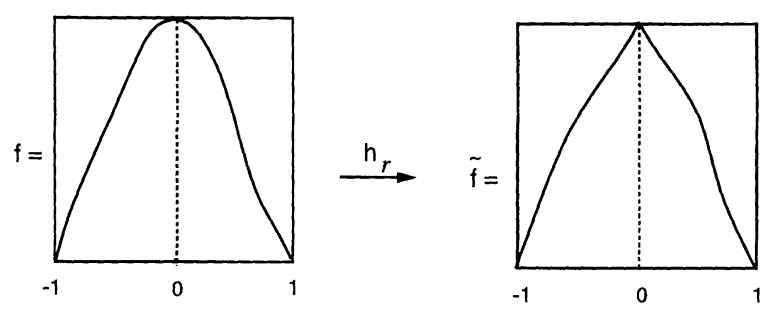

Fig. 1.

condition, and furthermore, any two such mappings $f$ and $g$ are topologically conjugate, that is, there is a homeomorphism $h$ of $[-1,1]$ such that $f \circ h=h \circ g$. In [3] (see[4]), we proved that $h$ is a quasisymmetric homeomorphism [1] provided $f$ and $g$ are both Ulam-von Neumann transformations which we will define in Sect. 3. Thus it is a Hölder continuous homeomorphism [1]. Usually $h$ is not smooth because $f$ and $g$ have a lot of smooth invariants, for example, all eigenvalues of $f$ at periodic points. For $f$ and $g$ are expanding circle endomorphisms, Shub and Sullivan [8] proved that if $h$ is absolutely continuous, then it is smooth. Similar work has been done by Herman [2] for circle diffeomorphisms and by de Llave and R. Moriyón in [7] for Anosov diffeomorphisms of a torus. All these are results for mappings without critical points. An important problem is to smoothly classify mappings with critical points. In this paper, we will smoothly classify Ulam-von Neumann transformations which are certain smooth self mappings $f$ of $[-1,1]$ with a unique power law singular point 0 and whose graph looks like those in Fig. 1.

Suppose $f$ is a Ulam-von Neumann transformation. The exponent of $f$ is a unique number $\gamma \geqq 1$ such that $f^{\prime}(x) /|x|^{\gamma-1}$ tends to non-zero numbers $B_{-}$and $B_{+}$as $x$ goes to 0 from the left and right, respectively. The asymmetry of $f$ is $A=B_{-} / B_{+}$which is the limit of $f^{\prime}(-x) / f^{\prime}(x)$ as $x$ decreases to 0 . The eigenvalue of $f$ at a periodic point of $p$ of period $n$ is $E_{p}=\left(f^{\circ n}\right)^{\prime}(p)$. We will prove that any two Ulam-von Neumann transformations are topologically conjugate (Theorem 1) in Sect. 3. And moreover, we will prove the following main result in Sect. 4.

Theorem 2. Suppose $f$ and $g$ are two Ulam-von Neumann transformations and $H$ is the conjugacy from $f$ to $g$. Then $H$ is a $C^{1+\varepsilon}$-diffeomorphism for some $0<\varepsilon \leqq 1$ if and only if the eigenvalues at all corresponding periodic points and the exponents and asymmetries of $f$ and $g$ are the same.

In order to study a Ulam-von-Neumann transformation $f$, we will use a change of metric similar to the one used by Ulam and von Neumann. The change of metric has singularities of the same type at the two boundary points -1 and 1 of the interval $[-1,1]$. It is universal in the sense that it does not depend on any particular mapping $f$, but only on the power law $|x|^{\gamma}$. Suppose $y=h_{\gamma}(x)$ is the corresponding change of coordinate on the interval $[-1,1]$. After this change of coordinate, $f$ becomes $\tilde{f}=h_{\gamma} \circ f \circ h_{\gamma}^{-1}$, which is smooth except at the point 0 . The mapping $\tilde{f}$ has nonzero derivative at every point except for a unique nondifferentiable point 0 . At zero, the left and right derivatives of $\tilde{f}$ exist and are positive and negative, respectively. A nice feature of the mapping $q(x)=1-2 x^{2}$ is that $\tilde{q}$ is expanding with Hölder continuous derivative, which implies that a certain binary tree of intervals associated with the dynamics of $\tilde{q}$ has bounded geometry 
(see $[3,4])$. The expanding property does not carry over to our more general setting but the bounded geometry does. This is our main idea to study Ulam-von Neumann transformations.

\section{Singular Change of Metric on an Interval}

Suppose $f$ is a piecewise $C^{1}$ self mapping of an interval $I$. A singular point $f$ is either a non-differentiable point or a differentiable point with zero derivative. There is a linear fractional transformation $H(x)=(a x+b) /(c x+d)$ maps $I$ onto the interval $[-1,1]$ homeomorphically such that $H \circ f \circ H^{-1}$ is a self mapping of $[-1,1]$. So we only need to consider a piecewise $C^{1}$ self mapping $f$ of the interval $[-1,1]$. A singular point $s$ is said to be power law if there is a real number $\gamma \geqq 1$ such that the limits

$$
\lim _{x \mapsto s^{-}} \frac{f^{\prime}(x)}{|x-s|^{\gamma-1}}=B_{-} \quad \text { and } \quad \lim _{x \mapsto s^{+}} \frac{f^{\prime}(x)}{|x-s|^{\gamma-1}}=B_{+}
$$

exist and are nonzero. Here $\gamma$ is called an exponent of $f$ and $A=B_{-} / B_{+}$is called an asymmetry of $f$. For example, $f$ has power law $|x|^{\gamma}$ at 0 if $f(x)=1-B_{-}|x|^{\gamma}$ for negative $x$ close to zero and $f(x)=1-B_{+}|x|^{\gamma}$ for positive $x$ close to zero. We will always make the following assumptions:

(1) $f$ is a piecewise $C^{1}$ self mapping of $[-1,1]$ with a unique power law singular point 0 ,

(2) $f \mid[-1,0]$ is $C^{1}$ and increasing, and $f \mid[0,1]$ is $C^{1}$ and decreasing, and

(3) $f$ maps 0 to 1 , and maps -1 and 1 to -1 (see Fig. 1).

Suppose $f$ is a self mapping satisfying (1), (2), and (3) and $\gamma>1$ is the exponent of $f$. Then $f$ is $C^{1}$ on $[-1,1]$ and 0 is a critical point, i.e., $f^{\prime}(0)=0$. We define the singular metric associated to $f$ to be

$$
d y=\frac{d x}{\left(1-x^{2}\right)^{\frac{\gamma-1}{\gamma}}}
$$

on $[-1,1]$. The corresponding change of coordinate on $[-1,1]$ is $y=h_{\gamma}(x)$, where

$$
h_{\gamma}(x)=-1+b \int_{-1}^{x} \frac{d x}{\left(1-x^{2}\right)^{\frac{\gamma-1}{\gamma}}}
$$

and $b$ is a number such that $h_{\gamma}(1)=1$. The representation of $f$ under the singular metric associated to $f$ is

$$
\tilde{f}=h_{\gamma} \circ f \circ h_{\gamma}^{-1} .
$$

Lemma 1. The mapping $\tilde{f}$ is continuous on $[-1,1]$, and $\tilde{f} \mid[-1,0]$ and $\tilde{f} \mid[0,1]$ are $C^{1}$-embeddings.

Proof. If $y$ is not one of 0,1 , and -1 , then $\tilde{f}$ is differentiable at $y$. Suppose $x$ is the preimage of $y$ under $h_{\gamma}$. By the chain rule,

$$
\tilde{f}^{\prime}(y)=\frac{f^{\prime}(x)\left(1-x^{2}\right)^{\frac{\gamma-1}{\gamma}}}{\left(1-(f(x))^{2}\right)^{\frac{\gamma-1}{\gamma}}} .
$$


Using this equation, we can get that $\tilde{f}^{\prime}(0-)$ and $\tilde{f}^{\prime}(0+)$ exist and equal nonzero numbers and that $\tilde{f}^{\prime}(-1)=\left(f^{\prime}(-1)\right)^{\frac{1}{\gamma}}$ and $\tilde{f}^{\prime}(1)=-\left|f^{\prime}(1)\right|^{\frac{1}{\gamma}}$.

Remark 1. The inverse of $h_{\gamma}$ is $C^{1}$. If the restrictions of $r_{f}=f^{\prime}(x) /|x|^{\gamma-1}$ to $[-1,0)$ and to $(0,1]$, and the restrictions of $f$ to $[-1,0]$ and to $[0,1]$ are $\alpha$-Hölder continuous for some $0<\alpha \leqq 1$, then the restrictions of $\tilde{f}$ to $[-1,0]$ and to $[0,1]$ are at least $C^{1+\alpha}$.

Lemma 2. Suppose $\tilde{f}$ is a continuous self mapping of $[-1,1]$. Assume 0 is its unique turning point, $\tilde{f}$ maps 0 to 1 and maps -1 and 1 to -1 and the restrictions of $\tilde{f}$ to $[-1,0]$ and to $[0,1]$ are $C^{1}$-embeddings. Then for every $\gamma>1, f=h_{\gamma}^{-1} \circ$ $\tilde{f} \circ h_{\gamma}$ is a $C^{1}$ mapping and has a unique power law $|x|^{\gamma}$ singular point 0 .

Proof. If $x$ is not one of 0,1 and -1 , then $f$ is differentiable at $x$. Suppose $y=h_{\gamma}(x)$. By the chain rule,

$$
f^{\prime}(x)=\frac{\tilde{f}^{\prime}(y)\left(1-\left(h_{\gamma}^{-1} \circ \tilde{f}(y)\right)^{2}\right)^{\frac{\gamma-1}{\gamma}}}{\left(1-\left(h_{\gamma}^{-1}(y)\right)^{2}\right)^{\frac{\gamma-1}{\gamma}}} .
$$

Using this equation, $f^{\prime}(-1)=\left(\tilde{f}^{\prime}(-1)\right)^{\gamma}$ and $f^{\prime}(1)=-\left|\tilde{f}^{\prime}(1)\right|^{\gamma}$, and the limits of $r_{f}(x)=f^{\prime}(x) /|x|^{r-1}$ as $x$ increases to zero and as $x$ decreases to zero exist and equal nonzero numbers.

Remark 2. The map $h_{\gamma}$ is $(1 / \gamma)$-Hölder continuous. If the restrictions of $\tilde{f}$ to $[-1,0]$ and to $[0,1]$ are $C^{1+\alpha}$ embeddings for some $0<\alpha \leqq 1$, then $f$ is $C^{1+\alpha / \gamma}$ and the restrictions of $r_{f}(x)=f^{\prime}(x) /|x|^{\gamma-1}$ to $[-1,0)$ and to $(0,1]$ are $\alpha / \gamma$-Hölder continuous.

Remark 3. For $\gamma=1$, let $\tilde{f}(x)=f(x)$ and $h_{1}(x)=x$.

\section{Ulam-von Neumann Transformations}

Suppose $f$ is a piecewise $C^{1}$ self mapping of the interval $[-1,1]$ satisfying (1), (2), and (3). Let $f_{0}$ and $f_{1}$ be the restrictions of $f$ to $[-1,0]$ and to $[0,1]$, respectively. Then $f_{0}$ and $f_{1}$ are both embeddings. Let $g_{0}$ and $g_{1}$ be the inverse of $f_{0}$ and $f_{1}$. For a finite string $w_{n}=i_{0} \cdots i_{n-1}$ of zeros and ones, let $g_{w_{n}}$ be the composition $g_{w_{n}}=g_{i_{0}} \circ \cdots \circ g_{i_{n-1}}$ of $g_{i_{0}}$ to $g_{i_{n-1}}$ and $I_{w_{n}}$ be the image of $[-1,1]$ under $g_{w_{n}}$. Suppose $\eta_{n, f}$ is the set of intervals $I_{w_{n}}$ for all finite strings $w_{n}$ of zeros and ones of length $n$. We call $\eta_{n, f}$ the $n^{\text {th }}$-partition of $[-1,1]$ by $f$. We use $\lambda_{n, f}$ to denote the maximum length of the intervals in $\eta_{n, f}$. The $n^{\text {th }}$-partition $\tilde{\eta}_{n, f}$ of $[-1,1]$ by $\tilde{f}$ and the maximum length $\tilde{\lambda}_{n, f}$ of the intervals in $\tilde{\eta}_{n, f}$ are defined similarly. (We will suppress $f$ if there is no confusion.)

Suppose $\Sigma=\prod_{n=0}^{\infty}\{0,1\}$ is the set of infinite sequences $w=i_{0} i_{1} \cdots$ of zeros and ones with product topology and $\sigma(w)=i_{1} \cdots$ is the shift map of $\Sigma$.

Lemma 3. If $\lambda_{n}$ tends to zero as $n$ goes to infinity, then there is a continuous mapping $h$ from $\Sigma$ onto $[-1,1]$ such that $f \circ h=h \circ \sigma$ on $\Sigma$. Moreover, $h$ is one-to-one except on a countable subset $\Gamma$ of $\Sigma$, and on $\Gamma, h$ is two-to-one.

Proof. For every $w=i_{0} i_{1} \cdots i_{n-1} \cdots$ in $\Sigma$ and an integer $n \geqq 0$, there is an interval $I_{w_{n}}$ in $\eta_{n}$ where $w_{n}=i_{0} \cdots i_{n-1}$. The interval $I_{w_{n+1}}=g_{w_{n+1}}([-1,1])$ is contained in 
$I_{w_{n}}=g_{w_{n}}([-1,1])$. So $I_{w}=\bigcap_{n=0}^{\infty} I_{w_{n}}$ is a non-empty set. Since $\lambda_{n}$ tends to zero as $n$ goes to infinity, $I_{w}$ contains only one point $x_{w}$. Let $h(w)=x_{w}$. It is a mapping from $\Sigma$ to $[-1,1]$.

Two points $w$ and $w^{\prime}$ are $n$-closed if their first $n$ digits are the same. Suppose $w=\cdots w_{n}$ and $w^{\prime}=\cdots w_{n}$ are $n$-closed. Then $x_{w}$ and $x_{w^{\prime}}$ are in $I_{w_{n}}$. This implies that $h\left(w^{\prime}\right)$ tends to $h(w)$ as $w^{\prime}$ goes to $w$. So $h$ is continuous.

The mapping $h$ is onto because $\bigcup_{w_{n} \in \eta_{n}} I_{w_{n}}=[-1,1]$ for all $n \geqq 0$. Let $\Gamma$ be the set of points $w$ which are preimages of endpoints $p \neq \pm 1$ of intervals of $\eta_{n}$ under $h$ for all $n \geqq 0$. Then $\Gamma$ is a countable subset. The mapping $h$ is one-to-one on $\Sigma \backslash \Gamma$ and two-to-one on $\Gamma$.

Definition 1. We say that the sequence of nested partitions $\left\{\eta_{n}\right\}_{n=0}^{\infty}\left(\right.$ or $\left.\left\{\tilde{\eta}_{n}\right\}_{n=0}^{\infty}\right)$ by $f($ or $\tilde{f})$ decreases exponentially if there are two constants $C>0$ and $0<\mu<1$ such that $\lambda_{n} \leqq C \mu^{n}$ (or $\left.\tilde{\lambda}_{n} \leqq C \mu^{n}\right)$ for all integers $n \geqq 0$.

Lemma 4. The sequence of nested partitions by $f$ decreases exponentially if and only if the sequence of nested partitions of $\tilde{f}$ decreases exponentially.

Proof. Because $h_{\gamma}$ is $(1 / \gamma)$-Hölder continuous and the inverse of $h_{\gamma}$ is $C^{1}$, one can easily see this lemma.

Definition 2. A self mapping $f$ of the interval $[-1,1]$ is said to be a Ulam-von Neumann transformation if it satisfy (1), (2), (3) in Sect. 2, and

(4) the restrictions of $\tilde{f}$ to $[-1,0]$ and to $[0,1]$ are $C^{1+\alpha}$ embeddings for some $0<\alpha \leqq 1$,

(5) the sequence of nested partitions $\left\{\tilde{\eta}_{n}\right\}_{n=0}^{\infty}$ by $\tilde{f}$ decreases exponentially.

Next lemma follows from Remark 1, Remark 2 and Lemma 4.

Lemma 5. A self mapping $f$ of the interval $[-1,1]$ satisfying (1), (2), and (3) in Sect. 2 is a Ulam-von Neumann transformation if and only if it also satisfies

(4) $f \mid[-1,0]$ and $f \mid[0,1]$ are $C^{1+\alpha}$ for some $0<\alpha \leqq 1$ and the restrictions of $r_{f}(x)=f^{\prime}(x) /|x|^{\gamma-1}$ to $[-1,0)$ and to $(0,1]$ are $\beta$-Hölder continuous for some $0<\beta \leqq 1$ and

(5)' the sequence $\left\{\eta_{n}\right\}_{n=0}^{\infty}$ of nested partitions by $f$ decreases exponentially.

Remark 4. The condition (4)' in Lemma 5 and $A=1$ are equivalent to the statement: $f(x)=F\left(-|x|^{\gamma}\right)$, where $F$ is a $C^{1+\alpha}$ diffeomorphism from $[-1,0]$ to $[-1,1]$ for some $0<\alpha \leqq 1$ (see [5]).

We give two examples of Ulam-von Neumann transformations.

Example 1. A self mapping $f$ of the interval $[-1,1]$ satisfying (1), (2), and (3) and the conditions (a) $f \mid[-1,0]$ and $f \mid[0,1]$ are $C^{3}$ mappings whose Schwarzian derivatives are non-positive and (b) $\left|f^{\prime}(-1)\right|>1$ and $\left|f^{\prime}(1)\right|>1$.

Remark 5. The Schwarzian derivative $S(f)$ of a function $f$ is, by definition,

$$
S(f)=\frac{f^{\prime \prime \prime}}{f^{\prime}}-\frac{3}{2}\left(\frac{f^{\prime \prime}}{f^{\prime}}\right)^{2} .
$$

The function $f$ is said to have non-positive Schwarzian derivative if $S(f)(x) \leqq 0$ for all $x$ in the domain of $f$. 
Example 2. A self mapping $f$ of the intervals [-1,1] satisfying (1), (2), and (3) and the conditions (i) $\tilde{f} \mid[-1,0]$ and $\tilde{f} \mid[0,1]$ are $C^{1+1}$ embeddings and (ii) all the periodic points of $f$ are expanding, that is, the absolute values of the eigenvalues of $f$ at all periodic points are greater than one.

The eigenvalue of $f$ at a periodic point $p$ of period $n$ of $f$ is $E_{f}(p)=$ $\left(f^{\circ n}\right)^{\prime}(p)$.

Remark 6. A function $g$ of an interval is said to be $C^{1+1}$ if its derivative $g^{\prime}$ is Lipschitz continuous, that is, there is a constant $C>0$ such that

$$
\left|g^{\prime}(x)-g^{\prime}(y)\right| \leqq C|x-y|
$$

for all $x$ and $y$ in the domain of $g$.

To prove that Examples 1 and 2 are Ulam-von Neumann transformations, one needs to check (5) in Definition 2 or (5)' in Lemma 5. This can be implied if $\tilde{f}$ or $f$ has bounded geometry [3,4]. In [3], we proved that $f$ has bounded geometry if it is a mapping in Example 1 and that $\tilde{f}$ has bounded geometry if it is a mapping in Example 2. Hence Examples 1 and 2 are Ulam-von Neumann transformations. Since it is not a main topic in this paper, we will not write down the proofs in detail. The reader may refer to [3] for the proofs.

Theorem 1. Any two Ulam-von Neumann transformations $f$ and $g$ are topologically conjugate.

Proof. From Lemma 3, there are two continuous mappings $h_{1}$ and $h_{2}$ from $\Sigma$ onto $[-1,1]$ such that $f \circ h_{1}=h_{1} \circ f$ and $g \circ h_{2}=h_{2} \circ g$. Here $h_{1}$ and $h_{2}$ on $\Sigma \backslash \Gamma$ are one-to-one. Thus $H=h_{1} \circ h_{2}^{-1}$ is defined on $[-1,1] \backslash h_{2}(\Gamma)$ and is uniformly continuous. It can be extended to a one-to-one continuous mapping from $[-1,1]$ onto itself. Similar argument can be applied to $H^{-1}=h_{2} \circ h_{1}^{-1}$. So $H$ is a homeomorphism of $[-1,1]$ and $f \circ H=H \circ g$.

Remark 7. Furthermore, $H$ is a quasisymmetric homeomorphism $[3,4]$. This implies that $H$ is Hölder continuous [1].

\section{Complete Smooth Invariants}

A $C^{1}$-invariant of a Ulam-von Neumann transformation $f$ is an object associated to $f$ which is the same for $f$ and for $H \circ f \circ H^{-1}$ whenever $H$ is an orientation preserving $C^{1}$-diffeomorphism. The conjugacy $H$ between two Ulam-von Neumann transformations $f$ and $g$ is usually not a diffeomorphism because $f$ has a lot of $C^{1}$ invariants, for example, the eigenvalue of $f$ at a periodic point, the exponent and asymmetry of $f$. An important question is that are these $C^{1}$ invariants complete? We give an affirmative answer to this question.

Theorem 2. Suppose $f$ and $g$ are two Ulam-von Neumann transformations and $H$ is the conjugacy from $f$ to $g$. Then $H$ is a $C^{1+\varepsilon}$-diffeomorphism for some $0<\varepsilon \leqq 1$ if and only if the eigenvalues at all corresponding periodic points and the exponents and asymmetries of $f$ and $g$ are the same.

Before to prove Theorem 2, we will prove some very useful lemmas. The first one is the well-known naive distortion lemma. 
Lemma 6. Suppose $g$ from an interval $I$ into the interval $[-1,1]$ is a $C^{1+\alpha}$ mapping for some $0<\alpha \leqq 1$ and $a_{0}=\inf _{x \in I}\left|g^{\prime}(x)\right|>0$. Let $b_{0}=\sup _{x \neq y \in I}\left(\mid g^{\prime}(x)-\right.$ $\left.g^{\prime}(y)|/| x-\left.y\right|^{\alpha}\right)<\infty$. Then for any two sequences $\left\{x_{i}\right\}_{i=1}^{n}$ and $\left\{y_{i}\right\}_{i=1}^{n}$ in $I$,

$$
\log \left(\prod_{i=1}^{n}\left|\frac{g^{\prime}\left(x_{i}\right)}{g^{\prime}\left(y_{l}\right)}\right|\right) \leqq \frac{b_{0}}{a_{0}} \sum_{i=1}^{n}\left|x_{i}-y_{i}\right|^{\alpha} .
$$

Proof. The proof of this lemma is easy for

$$
\begin{aligned}
\log \left(\prod_{i=1}^{n}\left|\frac{g^{\prime}\left(x_{i}\right)}{g^{\prime}\left(y_{i}\right)}\right|\right) & \leqq \sum_{i=1}^{n}|\log | g^{\prime}\left(x_{i}\right)|-\log | g^{\prime}\left(y_{i}\right)|| \\
& \leqq \sum_{i=1}^{n} \frac{1}{a_{0}}\left|g^{\prime}\left(x_{i}\right)-g^{\prime}\left(y_{i}\right)\right| \leqq \sum_{i=1}^{n} \frac{b_{0}}{a_{0}}\left|x_{i}-y_{i}\right|^{\alpha} .
\end{aligned}
$$

Lemma 7. A Ulam-von Neumann transformation $f$ is ergodic.

Proof. Let $m(\cdot)$ denote the Lebesgue measure. Suppose the exponent of $f$ is $\gamma \geqq 1$. Let $\tilde{f}$ be the representation of $f$ under the singular metric $d_{\gamma} y$. Suppose $X$ is a $f$-invariant subset of $[-1,1]$ and $m(X)>0$. Let $\tilde{X}=h_{\gamma}(X)$. Then it is a $\tilde{f}$-invariant subset of $[-1,1]$ and $m(\tilde{X})>0$.

Suppose $p$ is a Lebesgue density point of $\tilde{X}$. There is a nested sequence of intervals $I_{n}$ in $\tilde{\eta}_{n}$ containing $p$ such that

$$
\lim _{n \rightarrow \infty} \frac{m\left(I_{n} \cap \tilde{X}\right)}{m\left(I_{n}\right)}=1
$$

From Lemma 6, the chain rule, and (5) of Definition 2, there is a constant $C>0$ such that

$$
C^{-1} \leqq \frac{\left|\left(\tilde{f^{\circ}}\right)^{\prime}(x)\right|}{\left|\left(\tilde{f^{\circ n}}\right)^{\prime}(y)\right|} \leqq C
$$

for all $x$ and $y$ in $I_{n}$ and $n \geqq 1$. Thus

$$
\lim _{n \rightarrow \infty} \frac{m\left(\tilde{f}^{\circ n}\left(I_{n} \cap \tilde{X}\right)\right)}{m\left(\tilde{f}^{\circ n}\left(I_{n}\right)\right)}=1 .
$$

But $\tilde{f}^{\circ n}\left(I_{n}\right)=[-1,1]$ and $\tilde{f}^{\circ n}\left(I_{n} \cap \tilde{X}\right) \subset \tilde{X}$. So $m(\tilde{X})=m([-1,1])$. This implies that $m(X)=m([-1,1])$, i.e., $X$ has full measure. Hence $f$ is ergodic.

Suppose $f$ and $g$ are two Ulam-von Neumann transformations and $H$ is the conjugacy from $f$ to $g$, i.e., $f \circ H=H \circ g$. Suppose $\gamma_{f} \geqq 1$ and $\gamma_{g} \geqq 1$ are the exponents of $f$ and $g$. Then $\tilde{H}=h_{\gamma_{f}} \circ H \circ h_{\gamma_{g}}^{-1}$ is the conjugacy from $\tilde{f}$ to $\tilde{g}$, i.e., $\tilde{f} \circ \tilde{H}=\tilde{H} \circ \tilde{g}$. We say $\tilde{H}$ is absolutely continuous if it is non-singular with respect to the Lebesgue measure $m$, that is, $m(X)=0$ if and only if $m(\tilde{H}(X))=$ 0 . For example, if $\tilde{H}$ and $\tilde{H}^{-1}$ are both Lipschitz continuous, then $\tilde{H}$ and $\tilde{H}^{-1}$ are absolutely continuous. Suppose $p$ is a periodic point of $g$ of period $n$. Then $H(p)$ is a periodic point of $f$ of period $n$ too. The points $p$ and $H(p)$ are called corresponding periodic points.

Lemma 8. Suppose the eigenvalues of $f$ and $g$ at all corresponding periodic points are the same and $\gamma_{f}=\gamma_{g}$. Then $\tilde{H}$ is bi-Lipschitz. 
Proof. From the proof of Lemma 1 and the fact that $h_{\gamma} \mid(-1,1)$ is $C^{1}$, the eigenvalues of $\tilde{f}$ and $\tilde{g}$ at all corresponding periodic points are the same too.

From Lemma 6, the chain rule, and (5) of Definition 2, there is a constant $C>0$ so that

$$
C^{-1} \leqq \frac{\left|\left(\tilde{f}^{\circ n}\right)^{\prime}(x)\right|}{\left|\left(\tilde{f}^{\circ n}\right)^{\prime}(y)\right|} \leqq C
$$

for any $n>0$ and any $x$ and $y$ in $I \in \tilde{\eta}_{n, f}$, and

$$
C^{-1} \leqq \frac{\left|(\tilde{g} \circ n)^{\prime}(x)\right|}{\left|(\tilde{g} \circ n)^{\prime}(y)\right|} \leqq C
$$

for any $n>0$ and any $x$ and $y$ in $I \in \tilde{\eta}_{n, g}$.

Now for any interval $I$ in $\tilde{\eta}_{n, g}$, the ratio

$$
\frac{|\tilde{H}(I)|}{|I|}=\frac{\left|\left(\tilde{g}^{\circ n}\right)^{\prime}(\xi)\right|}{\left|\left(\tilde{f}^{\circ n}\right)^{\prime}(\tau)\right|}
$$

for some $\xi$ in $I$ and $\tau$ in $\tilde{H}(I)$ because both images of $I$ and $\tilde{H}(I)$ under the $\tilde{g}^{\circ}$ and $\tilde{f}^{\circ n}$ are $[-1,1]$. But $\tilde{g}$ on has a fixed point $p$ in $I$ and, from the condition in the lemma,

$$
\left(\tilde{g}^{\circ n}\right)^{\prime}(p)=\left(\tilde{f}^{\circ n}\right)^{\prime}(\tilde{H}(p)) .
$$

Therefore,

$$
C^{-2} \leqq \frac{|\tilde{H}(I)|}{|I|} \leqq C^{2}
$$

For any $x$ and $y$ in $[-1,1]$, the interval bounded by $x$ and $y$ can be written as a union of some intervals $\left\{I_{i}\right\}$ of $\left\{\tilde{\eta}_{n, g}\right\}_{n=0}^{\infty}$, where $\left\{I_{i}\right\}$ have pairwise disjoint interiors. Thus we have

$$
C^{-2} \leqq \frac{|\tilde{H}(x)-\tilde{H}(y)|}{|x-y|}=\frac{\sum_{i}\left|\tilde{H}\left(I_{i}\right)\right|}{\sum_{i}\left|I_{i}\right|} \leqq C^{2},
$$

which means that $\tilde{H}$ is bi-Lipschitz.

Proof of Theorem 2. The "only if" part can be proved from direct calculation. We will make an effort to prove the "if" part. From the previous lemma, $\tilde{H}$ is bi-Lipschitz. So it is absolutely continuous and orientation-preserving. Therefore $\tilde{H}^{\prime}(x)>0$ exists for a.e. $x$ in $[-1,1]$. Let $v=\log \tilde{H}^{\prime}$ a.e. on $[-1,1]$ and $\phi_{1}=$ $\log \left|\tilde{f}^{\prime} \circ \tilde{H}\right|$ and $\phi_{2}=\log \left|\tilde{g}^{\prime}\right|$ on $I_{0}$ and $I_{1}$. By the chain rule,

$$
\phi_{1}-\phi_{2}=v \circ \tilde{g}-v
$$

a.e. on $[-1,1]$. Our goal is to prove that $v$ can be extended to be a continuous function on $[-1,1]$.

Suppose $h$ is the semi-conjugacy from $(\sigma, \Sigma)$ to $(\tilde{g},[-1,1])$ (see Sect. 3). Then $h^{-1}(1)=100 \cdots$. There is a point $a$ in $\Sigma$ such that $\Phi=\left\{\sigma^{\circ n}(a) \mid n \geqq 0\right\}$ is dense in $\Sigma$. Define

$$
\tilde{u}\left(\sigma^{\circ n}(a)\right)=\sum_{i=0}^{n-1}\left(\phi_{1}\left(h\left(\sigma^{\circ i}(a)\right)\right)-\phi_{2}\left(h\left(\sigma^{\circ i}(a)\right)\right)\right)
$$


for $n \geqq 0$. Then $\tilde{u}$ is a function defined on $\Phi$ and

$$
\tilde{u} \circ \sigma-\tilde{u}=\phi_{1} \circ h-\phi_{2} \circ h
$$

on $\Phi$.

For any two different points $e_{1}=\sigma^{\circ n}(a)=b_{0} b_{1} \cdots$ and $e_{2}=\sigma^{\circ m}(a)=c_{0} c_{1} \cdots$ in $\Phi$. Assume $m>n$. There is an integer $k>0$ such that $b_{0} \cdots b_{k-1}=c_{0} \cdots c_{k-1}$ and $b_{k} \neq c_{k}$. Let $w_{k}=b_{0} \cdots b_{k-1}$ and

$$
\Lambda_{w_{k}}=\left\{w=i_{0} \cdots i_{n} \cdots \mid i_{0} \cdots i_{k-1}=w_{k}\right\} .
$$

Since $e_{2}=\sigma_{2}^{\circ(m-n)}\left(e_{1}\right), \sigma^{\circ(m-n)}$ has a fixed point $e_{3}$ in $\Lambda_{w_{k}}$. From the condition in the theorem and Eq. (1),

$$
\sum_{i=0}^{m-n-1}\left(\phi_{1}\left(h\left(\sigma^{\circ i}\left(e_{3}\right)\right)\right)-\phi_{2}\left(h\left(\sigma^{\circ i}\left(e_{3}\right)\right)\right)\right)=0 .
$$

Applying Lemma 6, the chain rule, and (5) of Definition 2, there are constants $C>0$ and $0<\mu<1$ such that

$$
\begin{aligned}
\left|\tilde{u}\left(e_{1}\right)-\tilde{u}\left(e_{2}\right)\right|= & \left|\sum_{i=n}^{m-1}\left(\phi_{1}\left(h\left(\sigma^{\circ i}(a)\right)\right)-\phi_{2}\left(h\left(\sigma^{\circ i}(a)\right)\right)\right)\right| \\
\leqq & \left|\sum_{i=0}^{m-n-1}\left(\phi_{1}\left(h\left(\sigma^{\circ(n+i)}(a)\right)\right)-\phi_{1}\left(h\left(\sigma^{\circ i}\left(e_{3}\right)\right)\right)\right)\right| \\
& +\left|\sum_{i=0}^{m-n-1}\left(\phi_{2}\left(h\left(\sigma^{\circ i}\left(e_{3}\right)\right)\right)-\phi_{2}\left(h\left(\sigma^{\circ(n+i)}(a)\right)\right)\right)\right| \leqq C \mu^{k} .
\end{aligned}
$$

This says that $\tilde{u}$ is uniformly continuous on $\Phi$. Thus it can be extended to a continuous function $u$ on $\Sigma$ and

$$
u \circ \sigma-u=\phi_{1} \circ h-\phi_{2} \circ h
$$

on $\Sigma$.

Suppose $h^{-1}(0)=\left\{a_{-}, a_{+}\right\}$, where $a_{-}=0100 \cdots$ and $a_{+}=1100 \cdots$. Then $\sigma\left(a_{ \pm}\right)=100 \cdots$ and, from Eq. (2),

$$
\begin{aligned}
& u(100 \cdots)-\lim _{w \rightarrow a_{-}} u(w)=\phi_{1}(0-)-\phi_{2}(0-), \\
& u(100 \cdots)-\lim _{w \rightarrow a_{+}} u(w)=\phi_{1}(0+)-\phi_{2}(0+) .
\end{aligned}
$$

By the condition $A_{f}=A_{g}$, we have

$$
\phi_{1}(0-)-\phi_{2}(0-)=\phi_{1}(0+)-\phi_{2}(0+) .
$$

So $\lim _{w \rightarrow a_{-}} u(w)=\lim _{w \rightarrow a_{+}} u(w)$. This implies that for any endpoint $p \neq \pm 1$ of an interval $I$ in $\tilde{\eta}_{n, g}$, let $h^{-1}(p)=\left\{w_{-}, w_{+}\right\}$, then

$$
\lim _{w \rightarrow w_{-}} u(w)=\lim _{w \rightarrow w_{+}} u(w)
$$


because $p$ is a preimage of 0 under some iterate of $\tilde{g}$. Hence $\chi(x)=u\left(h^{-1}(x)\right)$ is a well-defined and continuous function on $[-1,1]$, and, from Eq. (2),

$$
\chi \circ \tilde{g}-\chi=\phi_{1}-\phi_{2}
$$

on $[-1,1]$. Now, using Eq. (1), we can get that

$$
\chi \circ \tilde{g}-v \circ \tilde{g}=\chi-v
$$

a.e. on $[-1,1]$. Since $\tilde{g}$ is ergodic, $\chi(x)-v(x)$ equals a constant function $C$ a.e. on $[-1,1]$. Thus $v(x)$ can be extended to be a continuous function $v_{1}=\chi-C$.

Because $\tilde{H}$ is absolutely continuous,

$$
\tilde{H}(x)=-1+\int_{-1}^{x} e^{v_{1}(\xi)} d \xi .
$$

So $\tilde{H}$ is $C^{1}$.

Suppose $\tilde{f}$ and $\tilde{g}$ restricted to $[-1,0]$ and to $[0,1]$ are $C^{1+\alpha}$ for some $0<\alpha<1$. For any two points $x=x_{0}$ and $y=y_{0}$ in $[-1,1]$, let $x_{n}$ and $y_{n}$ in $[-1,0]$ be defined inductively so that they are the preimages of $x_{n-1}$ and $y_{n-1}$ under $\tilde{g}$. Then $\tilde{g}^{\circ}$ restricted to the interval bounded by $x_{n}$ and $y_{n}$ is a $C^{1+\alpha}$-diffeomorphism. Applying Lemma 6, the chain rule, and (5) of Definition 2, we have a constant $C>0$ such that

$$
\left|\sum_{i=0}^{n-1}\left(\phi_{1} \circ \tilde{g} \circ i\left(x_{n}\right)-\phi_{1} \circ \tilde{g} \circ i\left(y_{n}\right)\right)\right| \leqq C|\tilde{H}(x)-\tilde{H}(y)|^{\alpha}
$$

and

$$
\left|\sum_{i=0}^{n-1}\left(\phi_{2} \circ \tilde{g} \circ i\left(x_{n}\right)-\phi_{2} \circ \tilde{g} \circ i\left(y_{n}\right)\right)\right| \leqq C|x-y|^{\alpha}
$$

for any $x$ and $y$ in $[-1,1]$. From Eq. (3), we have

$$
v_{1} \circ \tilde{g}-v_{1}=\phi_{1}-\phi_{2}
$$

on $[-1,1]$. Equation (4) implies that

$$
v_{1}(x)-v_{1}\left(x_{n}\right)=\sum_{i=0}^{n-1}\left(\phi_{1} \circ \tilde{g} \circ i\left(x_{n}\right)-\phi_{2} \circ \tilde{g} \circ i\left(x_{n}\right)\right)
$$

and

$$
v_{1}(y)-v_{1}\left(y_{n}\right)=\sum_{i=0}^{n-1}\left(\phi_{1} \circ \tilde{g} \circ i\left(y_{n}\right)-\phi_{2} \circ \tilde{g} \circ i\left(y_{n}\right)\right) .
$$

Hence

$$
\begin{aligned}
& \left|v_{1}(x)-v_{1}(y)+v_{1}\left(y_{n}\right)-v_{1}\left(x_{n}\right)\right| \\
& \leqq\left|\sum_{i=0}^{n-1}\left(\phi_{1} \circ \tilde{g} \circ i\left(x_{n}\right)-\phi_{1} \circ \tilde{g} \circ i\left(y_{n}\right)\right)\right|+\left|\sum_{i=0}^{n-1}\left(\phi_{2} \circ \tilde{g} \circ i\left(x_{n}\right)-\phi_{2} \circ \tilde{g} \circ i\left(y_{n}\right)\right)\right| \\
& \quad \leqq\left(C \max _{x \in[-1,1]}\left|\tilde{H}^{\prime}(x)\right|+C\right)|x-y|^{\alpha} .
\end{aligned}
$$


Because $y_{n}-x_{n} \rightarrow 0$ as $n \rightarrow \infty$ and $v_{1}=\log \tilde{H}^{\prime}$ is uniformly continuous on $[-1,1]$, $v_{1}\left(y_{n}\right)-v_{1}\left(x_{n}\right) \rightarrow 0$ as $n \rightarrow \infty$. Thus

$$
\left|v_{1}(x)-v_{1}(y)\right| \leqq C^{\prime}|x-y|^{\alpha},
$$

where $C^{\prime}=C\left(\max _{x \in[-1,1]}\left|\tilde{H}^{\prime}(x)\right|+1\right)$. So $\tilde{H}$ is $C^{1+\alpha}$.

Now using $H=h_{\gamma}^{-1} \circ \tilde{H} \circ h_{\gamma}$ for $\gamma=\gamma_{f}=\gamma_{g}$, one can calculate directly that $H$ is $C^{1+\varepsilon}$ for $\varepsilon=\alpha / \gamma$. Similarly, one can prove that $H^{-1}$ is $C^{1+\varepsilon}$. It completes the proof of the "if" part.

Remark 8. From Theorem 2, the set of eigenvalues of $f$ at all periodic points, the exponent, and the asymmetry of $f$ are complete $C^{1}$ invariants. Moreover, from the proof of Theorem 2 and Eq. (4), for any $x$ in $[-1,1]$,

$$
v_{1}(x)=v_{1}(-1)+\sum_{i=0}^{\infty}\left(\phi_{1}\left(x_{i}\right)-\phi_{2}\left(x_{i}\right)\right)
$$

that is,

$$
\log \tilde{H}^{\prime}(x)=\log \tilde{H}^{\prime}(-1)+\sum_{i=0}^{\infty}\left(\log \left|\tilde{f}^{\prime}\left(\tilde{H}\left(x_{i}\right)\right)\right|-\log \left|\tilde{g}\left(x_{i}\right)\right|\right),
$$

where $x_{0}=x$ and $x_{i} \in[-1,0]$ is the preimage of $x_{i-1}$ under $\tilde{g}$. This equation gives a relation between $\tilde{H}$ and its derivative $\tilde{H}^{\prime}$. We can use it to study higher smoothness of $\tilde{H}$ if $\tilde{f}$ and $\tilde{g}$ are both $C^{k+\alpha}$ for some integer $k>1$ and some real number $0 \leqq \alpha \leqq 1$.

Acknowledgements. The author would like to thank Professor Dennis Sullivan for many insightful discussions.

\section{References}

1. Ahlfors, L.A.: Lectures on Quasiconformal Maps. Princeton, NJ: van Nostrand Company, 1966

2. Herman, M.R.: Sur la conjugaison différentiable des difféomorphismes du cercle á des rotations. Publ. Math. I.H.E.S., No. 49, 5-233 (1979)

3. Jiang, Y.: Generalized Ulam-von Neumann Transformations. Thesis (1990), Graduate Center of CUNY

4. Jiang, Y.: Geometry of geometrically finite one-dimensional maps. Commun. Math. Phys. 156, 639-647 (1993)

5. Jiang, Y.: Local normalization of one dimensional maps. IHES preprint, June (1989)

6. Jiang, Y.: Asymptotic differentiable structure on Cantor set. Commun. Math. Phys. 155, 503-509 (1993)

7. de la Llave, R., Moriyón, R.: Invariant for smooth conjugacy of hyperbolic dynamical system II. Commun. Math. Phys. 109, 369-378 (1987)

8. Shub, M., Sullivan D.: Expanding endomorphisms of the circle revisited. Ergod. Th \& Dynam. Sys. 5, 285-289 (1987) 
